\title{
Exploring the differential stages of the pigment metabolism by pre-harvest bagging and post-harvest ethylene de- greening of Eureka lemon peel
}

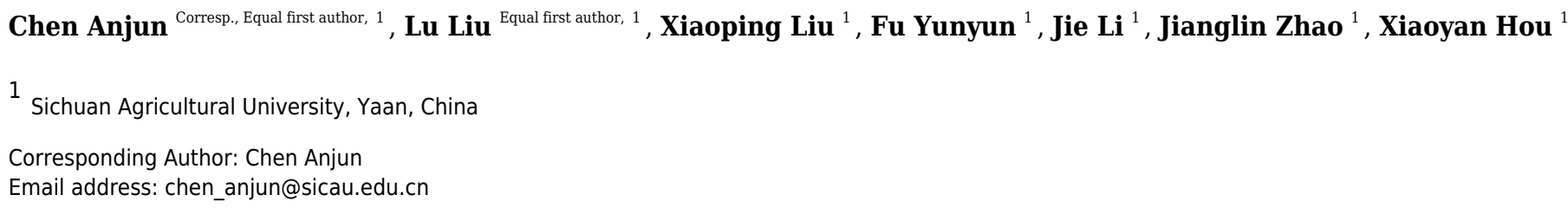

Pre-harvest bagging or post-harvest ethylene treatments on lemons are commonly applied to change the surface color from green to favorable yellow. In this study, the differential mechanisms of the pigment metabolism by the two treatments were investigated by pigments contents and related genetic expression. The results showed that both treatments reduced the number of chloroplasts and the content of chlorophyll. The differential expression of PSY1 and PSY2 were observed, causing the different accumulation of the main carotenoid phytoene content. The differential expression of NYC resulted in altered contents of chlorophyll $a$ and chlorophyll $b$, and further led to the difference in a* value. More interestingly, the degradation of chlorophyll uncovered the color of carotenoids, leading to the color changed from green to yellow. 
Exploring the differential stages of the pigment metabolism by pre-harvest bagging and postharvest ethylene de-greening of Eureka lemon peel

Abstract: Pre-harvest bagging or post-harvest ethylene treatments on lemons are commonly applied to change the surface color from green to favorable yellow. In this study, the differential mechanisms of the pigment metabolism by the two treatments were investigated by pigments contents and related genetic expression. The results showed that both treatments reduced the number of chloroplasts and the content of chlorophyll. The differential expression of PSY1 and PSY2 were observed, causing the different accumulation of the main carotenoid phytoene content. The differential expression of NYC resulted in altered contents of chlorophyll a and chlorophyll $b$, and further led to the difference in $a^{*}$ value. More interestingly, the degradation of chlorophyll uncovered the color of carotenoids, leading to the color changed from green to yellow.

Key words: chlorophyll degradation, carotenoid biosynthetic, genes, individual carotenoid contents

\section{Introduction}

As one of the third most important Citrus species after orange and mandarin (SernaEscolano et al, 2019), lemon (Citrus limon L. Burm.f.) is popular for its favorable flavor and natural ingredients like vitamins C (González-Molina et al, 2010) and phenolic compounds (Dong et al, 2019). Skin color is one of the key characteristics for citrus fruit, especially for lemons, because it largely affects the purchase intentions of consumers (Gao et al, 2019). Generally, customers prefer lemons with yellow color rather than green, however, lemons are 
usually harvested before fully ripe, when the fruit has the highest acid content, but the skin is still green. (Zhang and Zhou, 2019) One practical operation to obtain the yellow color is bagging during the fruit growing process. Pre-harvest bagging is commonly used as a physical protection method to reduce the mechanical damage, fruit cracking, and make the fruit dye evenly. (Yuan et al, 2019) It has also been widely used in other fruit such as grape, apple and kiwi (Guo et al, 2020; Yuan et al, 2019; Liao et al, 2019). Another way for the favorable yellow color is the ethylene treatment during the post-harvest process. Exogenous ethylene is regard as effective strategy to affect fruit color, and has been successfully applied to change the peel color from green to orange or yellow in navel orange and 'Eureka' lemon (Hu et al, 2018; Zhang et al, 2019). Previous studies on fruit de-greening were mostly focused on optimization of fruit bags materials, ethylene concentration, de-greening temperature and time (Liao et al, 2019; Jomori et al, 2014). However, the differential expression of the pigment metabolism by the two de-greening methods has not been reported yet.

The color of lemon is mainly determined by two main classes of pigments: chlorophylls and carotenoids: the former one is mainly responsible for green color, while the latter is mainly for yellow (Rodrigo et al, 2013). The biosynthesis and degradation of chlorophylls, as can be seen in Fig. 1, includes four stages: (1) synthesis of Mg-protoporphyrin IX from glutamic acid through a series of reactions, and then convert to chlorophyll a; (2) interconversion of chlorophyll a and chlorophyll b, also known as chlorine leaf plant cycle; (3) chlorophyll bound; and (4) degradation of chlorophyll (Tanaka and Tanaka, 2006).

Fig. 2 shows the biosynthesis pathways of carotenoids. In the general isoprenoid biosynthesis pathway, isopentenyl pyrophosphate (IPP), which is derived from glucose molecules, is the first relatively direct precursor ( $\mathrm{Lu}$ and $\mathrm{Li}, 2008)$. And though the isoprenoid pathway, IPP and dimethylallyl diphosphate (DMAPP) can be synthesized to geranylgeranyl diphosphate (GGPP), which is the central intermediate for carotenoids and other isoprenoid biosynthesis. Two molecules of GGPP can be condensed to phytoene (Li and Van, 2007), and subsequently, phytoene is dehydrogenated into ruthenium-carotene, and further dehydrogenated 
52 into lycopene (Issaacson et al, 2002). After that, there are two pathways downstream, affected by 53 the catalysis of different enzymes. In the first pathway, $\alpha$-carotene is synthesized by lycopene $\varepsilon$ 54 cyclase (LCYe) and lycopene $\beta$-cyclase (LYCb1), and further generates lutein (Nisar et al, 2015), 55 which is the most abundant carotenoid found in plant leaf tissue. As can be seen, LCYe plays a 56 key role in determining the ratio of $\beta$-carotene to $\alpha$-carotene (Harjes et al, 2008).

57 The biosynthesis and biodegradation of carotenoids and chlorophylls have been comprehensively investigated by classical genetic and molecular genetic analysis in some plants like pea and broccoli (Parveen et al, 2019; Luo et al, 2019). But it is still lack of studies in lemon. Although pre-harvest bagging and post-harvest ethylene de-greening treatments can help change the lemon color from green to a favorable yellow, the gene expressions and metabolites of these two methods were likely to be different. The present study was therefore conducted to explore the differential mechanisms of the chlorophyll degradation and carotenoid biosynthesis in preharvest bagging and post-harvest ethylene de-greening of lemon peel.

2 materials and methods

\subsection{Fruit materials}

Eureka lemon (Citrus limon(L.) Burm.f.) trees were cultivated in a 12-year-old lemon orchard located at Anyue County, Sichuan Province, China. In August of 2018, young fruit was bagged with paper bags (Sichuan Meishan Guanrong Bag Making Co.,Ltd.). (Wen, 2009 )The Bagged and non-bagged fruit was grown under the same cultural practice. The fruit with similar quality (TSS > 7.5) and weight between $140 \mathrm{~g}$ and $180 \mathrm{~g}$ was harvested at 2 November 2018. 
2.2 Ethylene treatment and storage

\subsection{Fruit surface color observation}

\subsection{Spectrophotometric determination of chlorophyll and total carotenoid content}

Chlorophyll and total carotenoid were extracted according to the method reported by Yuan et al, 2017. Briefly, $1.0 \mathrm{~g}$ peel was grounded to fine powders in liquid nitrogen. $10 \mathrm{~mL}$ acetone containing $0.01 \%$ BHT (2,6-di-tert-butylmethylphenol) was added, followed by centrifugation at $4,500 \times \mathrm{g}$ for $10 \mathrm{~min}$ at $4{ }^{\circ} \mathrm{C}$. The supernatant was collected and the residue was extracted again by the same procedure until colorless. The supernatants were mixed and were adjusted to $25 \mathrm{~mL}$ with acetone containing $0.01 \%$ BHT. The pigments contents were calculated based on the absorbance at 470, 645 and $662 \mathrm{~nm}$, respectively. 
2.5 LC MS/MS analysis of carotenoids

101

After harvesting, the three group of lemon peels were frozen in liquid nitrogen, and stored at -

103

104

$80{ }^{\circ} \mathrm{C}$ until use. $100 \mathrm{mg}$ sample was grounded into powder in liquid nitrogen, and extracted with a solution of n-hexane: acetone: ethanol at 2:1:1 (V/V/V). The extracts were vortexed for $30 \mathrm{~s}$, an ultrasound-assisted extraction was carried out for $20 \mathrm{~min}$ at room temperature, and then the extracts centrifugated at $10,000 \times \mathrm{g}$ for $5 \mathrm{~min}$. The supernatants were collected and the residue was extracted again by the same procedure. The supernatants were collected and evaporated to dryness under nitrogen gas flow. $200 \mu \mathrm{L} 75 \%$ methanol was added to reconstitute the solution. The supernatant was collected for LC-MS analysis.

The LC MS/MS analysis of carotenoids was performed by Metware Biotechnology Co., Ltd. (Wuhan, China). A LC-ESI-MS/MS system (UHPLC, ExionLCTM AD; MS, Biosystems 6500 Triple Quadrupole) was used. The analytical conditions were as follows: HPLC: YMC C30 column (3 um, 2 mm*100 mm); solvent system: acetonitrile : methanol (3:1, V/V with $0.01 \%$ BHT, eluent A) and methyl tert-butyl ether with $0.01 \%$ BHT (eluent B); gradient program: 85:15 A:B at $0 \min , 75: 25 \mathrm{~A}: \mathrm{B}$ at $2 \mathrm{~min}, 40: 60 \mathrm{~A}: \mathrm{B}$ at $2.5 \mathrm{~min}, 5: 95 \mathrm{~A}: \mathrm{B}$ at $3 \mathrm{~min}, 85: 15 \mathrm{~A}: \mathrm{B}$ at $6 \mathrm{~min}$; flow rate: $0.8 \mathrm{~mL} \mathrm{~min}{ }^{-1}$; temperature: $28{ }^{\circ} \mathrm{C}$; injection volume: $5 \mathrm{uL}$. The effluent was alternatively connected to an ESI-triple quadrupole-linear ion trap (Q TRAP)-MS.

API6500QTRAP LC/MS/MS System, equipped with an APCI Turbo lon-Spray interface, was operated in a positive ion mode and controlled by Analyst 1.6.3 software (AB Sciex). The APCI source operation parameters were as follows: ion source: turbo spray; source temperature: $350^{\circ} \mathrm{C}$; curtain gas (CUR): 25.0 psi; the collision gas (CAD): medium. DP and CE for individual MRM transitions were done with further DP and CE optimization. (Petry and Mercadante, 2018) 
124

125

126

127

2.6 RNA extraction and RNA-Seq

RNA extraction and RNA-Seq were conducted by Pacino Biotech Co.,Ltd. (Shanghai, China). The original off-machine data was filtered by removing the reads with a length of less than $50 \mathrm{bp}$ and a sequence average quality below Q20. The obtained high-quality sequence was spun-headed to a transcript sequence. The transcripts were clustered, the longest transcript was selected as Unigene. Unigene of each sample was obtained to investigate the expression difference analysis, enrichment analysis, cSNP and InDel analysis, including GO, KEGG, eggNOG, SwissProt, Pfam annotation and ORF prediction, SSR prediction, etc.

\subsection{Real-time quantitative PCR(RT-qPCR) analysis}

The primers for corresponding sequences were designed according to literatures and were listed in Table 1. CitActin was used as the reference gene. qRT-PCR was performed using TIB8600 (Taipu Biosciences Co., Ltd.). Each reaction contained $10 \mu \mathrm{L} 2 \times$ SYBR real-time PCR premixture, $0.4 \mu \mathrm{L}$ PCR forward primer $(10 \mu \mathrm{M}), 0.4 \mu \mathrm{L}$ PCR reverse primer $(10 \mu \mathrm{M}), 1 \mu \mathrm{L}$ cDNAs, and $\mathrm{RNase}$ free $\mathrm{ddH}_{2} \mathrm{O}$ to a final volume of $20 \mu \mathrm{L}$. The PCR conditions were $95{ }^{\circ} \mathrm{C}$ for $15 \mathrm{~min}$, followed by 40 cycles of $95{ }^{\circ} \mathrm{C}$ for $15 \mathrm{~s}$ and $60{ }^{\circ} \mathrm{C}$ for $30 \mathrm{~s}$. Three biological replicates were performed, and the mean values were used for RT-qPCR analysis. The relative expression of the genes was calculated according to the method of $2^{-\Delta \Delta \mathrm{Ct}}$.

\subsection{Statistical analysis}


147 Three biological replicates $(n=3 \times 3)$ were performed for all treatments. The data were analyzed 148 with SPSS 16.0 and Excel 2013, and the results were expressed as mean values with standard 149 deviation (SD). The difference was considered to be significant at $\mathrm{P}<0.05$.

3 Results

152

3.1 Fruit surface color changes

The effects of pre-harvest bagging and post-harvest ethylene treatment on lemon surface color were shown in Fig. 3. It can be seen that, after the treatments, the color of lemon peel was changed from green to yellow. The L*, a*, b* color parameters (Fig. 4) was corresponded to the visual observation. The $\mathrm{a}^{*}$ values of ethylene and bagged fruit were significantly higher than that of control. Control and ethylene treated fruit showed lower $\mathrm{L}^{*}$ value than bagging group. There were no significant changes in $b^{*}$ value for the three groups.

As shown in Fig. 5, the chlorophyll a and chlorophyll b content in bagging and ethylene fruit were significantly lower than that in control $(\mathrm{P}<0.05)$, while for the carotenoid content, ethylene treated fruit were significantly higher than bagging group, and control was the lowest one $(\mathrm{P}<$ 0.05). These results indicated that the ethylene and bagging treatment could degrade the 168 chlorophyll a and chlorophyll b content, and improve the carotenoid synthesis. 
Table 2 showed the carotenoid composition by different treatments. The results showed that

bagging and ethylene exhibited different effects on the accumulation of individual carotenoids. Bagging and ethylene treatment sharply increased the content of phytoene and capsanthin $(\mathrm{P}<$ 0.05), but decreased the content of lutein, $\alpha$-carotene, $\beta$-carotene and other substances $(\mathrm{P}<0.05)$. phytoene. The content of $\gamma$-carotene, phytoene and capsanthin in the ethylene group were obviously lower than that in the bagging group, whereas the content of lutein, neoxanthin, zeaxanthin, violaxanthin and antheraxanthin and capsorubin were the opposite.

3.4 Screening carotenoid biosynthesis and chlorophyll degradation related genes in the transcriptome

The genes involved in chlorophyll degradation and carotenoid biosynthesis were analyzed. 22 genes that encoded enzymes in the carotenoid biosynthetic and chlorophyll metabolic pathway were screened in lemon transcriptome: CHLH, NYC, Chlase, Pao, SGR, CAB1, CAB2, RCCR, PSY1, PSY2, PDS, ZDS, CRTISO, LCYb1, LCYb2, CHYB, ZEP, VDE, CCD1, NCED1, NCED2, NCED3, LCYe. The heat map of the carotenoid biosynthetic and chlorophyll metabolic related genes was drawn according to FPKM in Fig. 6. It can be seen that control and ethylene groups were more closely related than bagging group. PSY1, RCCR, CHLH, CCD1, SGR, NCED1, Chlase, ZDS, Pao, LCYE and CAB2 were closely related in a cluster, while the other genes were clustered in another group. 
194

195

196

197

198

199

200

201

202

203

204

205

206

207

208

209

210

211

212

213

214

215

216

217

218

In this transcriptome, two genes (PSY1 and PSY2) in ethylene group recorded a more expression than that in control and bagging groups. Furthermore, nine genes (CHYB, NCED3, NCED2, CRTISO, PDS, LCYB2, VDE, NYC) in bagging group expressed more than that in control and ethylene groups. And eleven genes (RCCR, CHLH, CCD1, SGR, NCED1, Chlase, ZDS, Pao, LCYE, CAB2, CAB1) in control group expressed more than that in ethylene and bagging group. Except for $\mathrm{NYC}$, the expression of the chlorophyll metabolism pathway genes (CHLH, Chlase, Pao, SGR, CAB1, CAB2, RCCR) were down-regulated after the peel turned yellow.

3.5 RT-qPCR analysis of transcription levels of chlorophyll and carotenoid degradation pathway genes.

As shown in Table 3, the genetic expressions of 22 key carotenoid biosynthetic and chlorophyll degradation pathway genes in different groups were studied using RT-qPCR analysis. The expression of CHLH, Chlase, Pao, CAB1, CAB2 and RCCR in bagging and ethylene groups were lower than that in control group. All of the expression of the chlorophyll degradation related genes, except RCCR, in bagging group were higher than that in ethylene group. NYC was up-regulated after pre-harvest bagging treatment. PSY gene is a multi-gene family, the present paper studied two members of CitPSY1 and CitPSY2. In the carotenoid biosynthetic pathway, the expression of PSY1, PDS, ZDS, CRTISO, LCYb2, CHYB, ZEP, VDE, CCD1, NCED1, NCED2, NCED3 and LCYe in ethylene group were lower than that in control group. The expression of all genes, except PSY2, in ethylene group were lower than that in bagging group. The results also showed that PSY1, CRTISO, VDE, NCED1, NCED2 and NCED3 were upregulated after bagging treatment. 
219

220

221

222

223

224

225

226

227

228

229

230

231

232

233

234

235

236

237

238

239

240

241

242

243

33

\section{Discussion}

The surface color of Citrus fruit is one of the most important quality characteristics that is a decisive factor for consumer acceptance (Rodrogo et al, 2013). Pre-harvest bagging treatment and post-harvest ethylene de-greening treatment can help change the lemon color from green to favorable yellow. Similarly as many other fruit, like kiwi and 'Valencia Delta' orange (Zhang et al, 2018; Pereira et al, 2016), the color of lemon peel is highly related to the changes of chlorophyll and carotenoid content (Yuan et al, 2017). The present study showed that chlorophyll a and chlorophyll b contents were declined after the yellowing process. The content of chlorophyll a in bagging group was higher than that in ethylene group, whereas the content of chlorophyll b was just the opposite. Although the bagging and ethylene fruits still contained ca. $50 \%$ of the Chlorophyll of the control treatment. The ratio of chlorophyll to carotenoids was 9.39 in the control group, 2.79 in the bagging group, and 2.03 in the ethylene group. The reason is not clear and need further study.

NYC protein has chlorophyll $\mathrm{b}$ reductase activity in vitro, and regulate the thylakoid membrane degradation (Cheng et al, 2012). In this study, NYC was up-regulated after preharvest bagging treatment, leading to higher degradation of chlorophyll $\mathrm{b}$ to chlorophyll $\mathrm{a}$. This phenomenon explained the reason why the bagging group recorded higher chlorophyll a content but lower chlorophyll b content than ethylene group. Except for $\mathrm{NYC}$, the expression of the chlorophyll metabolism pathway genes (CHLH, Chlase, Pao, SGR, CAB1, CAB2, RCCR) were down-regulated after the peel turned yellow. This result was accord to the other study in mandarin fruit (Yuan et al, 2017).

Bagging and ethylene treated lemons showed yellower color than control, and recorded higher 
244

245

246

247

248

249

250

251

252

253

254

255

256

257

258

259

260

261

262

263

264

265

266

267

268

269

270

content of phytoene and capsanthin, but lower content of lutein, $\alpha$-carotene, $\beta$-carotene and other substances than control group, suggesting that the yellow color of Eureka lemon may be mainly derived from phytoene. This could be primarily explained by the high expression of PSY and the low expression of the desaturase PDS (Kato et al, 2004). In the "green" lemon peel, the colored carotenoids were main lutein, which little influenced the yellow color of the lemon peel. Although this pigment was found in high quantities, the degradation pattern was similar as chlorophylls. When the peel was green, the concentration of lutein reached values up to $15.62 \mu \mathrm{g}$ $\mathrm{g}^{-1}$, but its color was masked by the chlorophylls. However, when they degraded, a decrease in lutein was also found, indicating that this pigment barely contributed to the color of lemon peel (Conesa et al, 2019). During the coloration process, the rapid loss of the chlorophyll played a leading role for the shift of fruit color from green to yellow, despite the content of the carotenoids (lutein, $\alpha$-carotene, $\beta$-carotene, et al.) decreased in the lemon peel (Shen et al, 2019).

The synthesis of carotenoid in the bagging and ethylene groups was analyzed based on the genetic expression and the changes of carotenoids content. The carotenoid levels were shown to be dependent on the PSY expression, which was the first rate-limiting enzyme in the carotenoid biosynthetic pathway presented in many plants such as tomato, apple, carrot, papaya and pepper (Fu et al, 2018; Heng et al, 2019; Fantini et al, 2013; Ampomah-Dwamena et al, 2015; Fuentes et al, 2012; Shen et al, 2019; Wei et al, 2019), it plays an important role in controlling the metabolism of the carotenoids. PSY1 is chromoplast-specific and its expression is extremely high during ripening stage of fruit, while PSY2 acts predominantly in chloroplast-containing tissues and does not have a contribution to the production of carotenoid in fruit (Cao et al, 2019). In our study, PSY1 was up-regulated after bagging treatment, and the expression of PSY2 in ethylene group was higher than that in bagging group. This phenomenon could explain why the phytoene content in bagging group was higher than that in ethylene group. Violaxanthin deepoxidase (VDE) and zeaxanthin epoxidaze (ZEP) are the two enzymes in the xanthophyll cycle. In excess light conditions, VDE catalyzes the conversion of violaxanthin to zeaxanthin via 
271 antheraxanthin, whereas ZEP catalyzes the reverse reaction (Shen et al, 2019). LCYE plays a 272 decisive role in the ratio of $\alpha$-carotene and $\beta$-carotene. The present study showed that high LCYE 273 expression resulted in high luteincontent, which was consistent with the study in marigold 274 (Cheng et al, 2019). The expression of the LCYE was lowered in both the bagging and ethylene groups. These results revealed that even though both pre-harvest bagging and post-harvest ethylene treatment could help change the lemon color from green to yellow, the mechanisms of

277 the two treatments were largely different.

278

279

280

281

282

283

284

285

286

287

288

289

290

291

292

293

294

295

\section{Conclusions}

This work mainly compared the differential mechanisms of chlorophyll degradation and carotenoid biosynthesis by pre-harvest bagging and post-harvest ethylene de-greening treatments, The results showed that both de-greening treatments can help to change the lemon peel color from green to favorable yellow. The expression of key genes and pigments contents in the lemon peel during the yellowing process revealed that carotenoid biosynthetic and chlorophyll degradation pathway by the two treatments were different. Our results clearly showed that the differential expression of PSY1 and PSY2 caused a difference in the main carotenoid phytoene by two treatments. The differential expression of NYC resulted in different contents of chlorophyll a and chlorophyll b, which showed a difference in $a^{*}$ value. Like other yellow citrus, the degradation of chlorophyll, which made the color of carotenoids appear, was the main reason for the changes of the lemon peel color from green to yellow.

\section{Availability of data and materials}

The RNA sequencing data were submitted to the Sequence Read Archive (SRA) of the NCBI database, as the BioProject ID PRJNA606613

(https://www.ncbi.nlm.nih.gov/sra/PRJNA606613). 
297

298

299

300

301

302

303

304

305

306

307

308

309

310

311

312

313

314

315

316

317

318

319

320

321

322

323

324

Ampomah-Dwamena, C., Driedonks, N., Lewis, D., Shumskaya, M., Chen, X.Y., Wurtzel, E. T., Espley, R. V., Allan, A. C. (2015) The Phytoene synthase gene family of apple (Malus x domestica) and its role in controlling fruit carotenoid content. BMC plant biol, 15(1): 185.

Cao, H. B., Luo, H. M., Yuan, H., Eissa, M.A., Thannhauser, T. W., Welsch, R., Hao, Y. J., Cheng, L. L., Li, L. (2019) A neighboring aromatic-aromatic amino acid combination governs activity divergence between tomato phytoene synthases. Plant Physiol, 180(4): 1988-2003.

Cheng, Y. D., Dong, Y., Yan, H. B., Ge, W. Y., Shen, C. G., Guan, J. F., Liu, L. Q., Zhang, Y. Y. (2012) Effects of 1-MCP on chlorophyll degradation pathway-associated genes expression and chloroplast ultrastructure during the peel yellowing of Chinese pear fruits in storage. Food chem, 135(2): 415-422.

Cheng, X., Zhao, X. Z., Huang, C. L., Zhang, X. H., Lyu, Y. M. (2019) Lutein content in petals and leaves of marigold and analysis of lutein synthesis gene expression. Acta Physiol Plant, 41(7): 128.

Conesa, A., Manera, F. C., Brotons, J. M., Fernandez-Zapata, J. C., Simon, I., Simon-Grao, S., Alfosea-simon, M., Martinez Nicolas, J. J., Valverde, J. M., Garcia-Sanchez, F. (2019) Changes in the content of chlorophylls and carotenoids in the rind of Fino 49 lemons during maturation and their relationship with parameters from the CIELAB color space. Sci Hortic, 243: 252-260.

Dong, X. Y., Hu, Y, Li, Y, Zhou, Z. Q. (2019) The maturity degree, phenolic compounds and antioxidant activity of Eureka lemon [Citrus limon (L.) Burm. f.]: A negative correlation between total phenolic content, antioxidant capacity and soluble solid content. Sci Hortic, 243: 281-289.

Fantini, E., Falcone, G., Frusciante, S., Giliberto, L., Giuliano, G. (2013) Dissection of tomato lycopene biosynthesis through virus-induced gene silencing. Plant physiol, 163(2): 986-998.

Fuentes, P., Pizarro, L., Moreno, J. C., Handford, M. (2012) Light-dependent changes in plastid differentiation influence carotenoid gene expression and accumulation in carrot roots. Plant mol biol, 79(1-2): 47-59.

Fu, X.M., Cheng, S. H., Feng, C., Kang, M., Huang, B. Z., Jiang, Y. M., Duan, X. W., Grierson, D., Yang, Z. Y. (2018) Lycopene cyclases determine high $\alpha$-/ $\beta$-carotene ratio and increased carotenoids in bananas ripening at high temperatures. Food chem, 283: 131-140.

Gao, Y., Liu, Y., Kan, C. N., Chen, M., Chen, J. Y. (2019) Changes of peel color and fruit quality in navel orange fruits under different storage methods. Sci Hortic, 256: 108522.

Guo, S. H., Xu, T. F., Shi, T. C., Jin, X. Q., Feng, M. X., Zhao, X. H., Zhang, Z. W., Meng, J. F.,(2020) 
Cluster bagging promotes melatonin biosynthesis in the berry skins of Vitis vinifera cv. Cabernet Sauvignon and Carignan during development and ripening. Food chem 305: 125502.

González-Molina, E., Domínguez-Perles, R., Moreno, D. A., García-Viguera, C. (2010) Natural bioactive compounds of Citrus limon for food and health. J pharmaceut biomed, 51(2): 327-345.

Harjes, C. E., Rocheford, T. R., Bai, L., Brutnell, T. P., Kandianis, C.B., Sowinski, S. G., Yan, J. (2008) Natural genetic variation in lycopene epsilon cyclase tapped for maize biofortification. Science, 319(5861): 330-333.

Heng, Z., Sheng, O., Huang, W. J., Zhang, S., Fernie, A. R., Motorykin, L., Kong, Q., Yi, G. J., Yan, S. J. (2019) Integrated proteomic and metabolomic analysis suggests high rates of glycolysis are likely required to support high carotenoid accumulation in banana pulp. Food Chem, 125016.

Hu, Y. Y., Wang, G. H., Pan, S. Y., Wang, L. F. (2018) Influence of ethylene and ethephon treatments on the peel color and carotenoids of Gannan Newhall navel orange during postharvest storage. $\mathrm{J}$ food biochem, 42(5): e12534.

Isaacson T., Ronen G., Zamir D., Hirschberg J. (2002) Cloning of tangerine from tomato reveals a carotenoid isomerase essential for the production of $\beta$-carotene and xanthophylls in plants. Plant Cell, 14(2): 333-342.

Jomori, M. L. L., Sasaki, F. F. C., Berno, N. D., Gimenes, L. C., Kluge, R. A. (2014) Degreening and cold storage of 'Murcott'tangor as function concentration and exposure time to ethylene. Semina: Ciências Agrárias 35(2): 825-834.

Kato, M., Ikoma, Y., Matsumoto, H., Sugiura, M., Hyodo, H., Yano, M. (2004) Accumulation of carotenoids and expression of carotenoid biosynthetic genes during maturation in citrus fruit. Plant Physiol 134(2): 824-837.

Lu, S., Li, L. (2008) Carotenoid metabolism: biosynthesis, regulation, and beyond. J Integr Plant Biol, 50(7): 778-785.

Luo, F., Cheng, S. C., Cai, J. H., Wei, B. D., Zhou, X., Zhou, Q., Zhang, Y., B., Ji, S. J. (2019). Chlorophyll degradation and carotenoid biosynthetic pathways: gene expression and pigment content in broccoli during yellowing. Food chem, 297: 124964.

Li, L., Van, E. J. (2007) Metabolic engineering of carotenoid accumulation by creating a metabolic sink. Transgenic res, 16(5): 581-585.

Liao, G. L., He, Y. Q., Li X. S., Zhong, M., Huang, C. H., Yi, S. Y., Liu, Q., Xu, X. B. (2019) Effects of bagging on fruit flavor quality and related gene expression of AsA synthesis in Actinidia eriantha. Sci 
Hortic 256: 108511.

Nisar, N., Li, L., Lu, S., Khin, N. C., Pogson, B. J. (2015) Carotenoid metabolism in plants. Mol plant, 8(1): 68-82.

Parveen, N., Shakeel, A., Malik, T. A., Awan, F. S. (2019) genetic studies for improving seed yield and quality traits including carotenoids, chlorophyll and protein contents in pea (pisum sativum L.). Pak J Bot, 51(6): 2105-2110.

Pereira, G. S., Machado, F. L. C., Costa, J. (2016) Quality of 'Valencia Delta'orange after degreening and coating with wax. Rev Bras Eng Agr Amb, 20(10): 936-940.

Petry, F. C. and Mercadante, A. Z. (2018) New method for carotenoid extraction and analysis by HPLC-DAD MS/MS in freeze-dried Citrus and Mango pulps. J Brazil Chem Soc. 1:205-215.

Serna-Escolano, V., Valverde, J. M., García-Pastor, M. E., Valero, D., Castillo, S., Guillen, F., Martinezromero, D., Zapata, P, Serrano.M. (2019) Pre - harvest methyl jasmonate treatments increase antioxidant systems in lemon fruit without affecting yield or other fruit quality parameters. J Sci Food Agr.99(11): 5035-5043.

Shen, Y. H., Yang, F. Y., Lu, B. G., Zhao, W. W., Jiang, T., Xiao, J. C., Ming, R. (2019) Exploring the differential mechanisms of carotenoid biosynthesis in the yellow peel and red flesh of papaya. BMC genomics, 20(1): 49.

Tanaka, A., Tanaka, R. (2006) Chlorophyll metabolism. Curr Opin plant biol , 9(3): 248-255.

Wei, F., Fu, M., Li, J., Yang, X. M., Chen, Q. Y., Tian S. P. (2019) Chlorine dioxide delays the reddening of postharvest green peppers by affecting the chlorophyll degradation and carotenoid synthesis pathways. Postharvest Bio Tec, 156: 110939.

Wen, S. (2009) Application of Techniques on Citrus Bagging culture. Academic Periodical of Farm Products Processing, 7.

Yuan, Z. Y., Deng, L. L., Yin, B. F., Yao, S. X., Zeng, K. F. (2017) Effects of blue LED light irradiation on pigment metabolism of ethephon-degreened mandarin fruit. Postharvest bio tec, 134: 45-54.

Yuan, G. P., Bian, S. X., Han, X. L., He, S. S., Liu, K., Zhang, C. X., Cong, P. H. (2019) An Integrated Transcriptome and Proteome Analysis Reveals New Insights into Russeting of Bagging and Non-Bagging “Golden Delicious” Apple. Int J mol Sci 20(18): 4462.

Zhang, J. Y., Pan, D. L., Jia, Z. H. Wang, T., Wang, G., Guo, Z. R. (2018) Chlorophyll, carotenoid and vitamin 
384 C metabolism regulation in Actinidia chinensis' Hongyang'outer pericarp during fruit development. Plos 385 one, 13(3): e0194835.

386 Zhang, P. Y., Zhou, Z. Q. (2019) Postharvest ethephon degreening improves fruit color, flavor quality and 387 increases antioxidant capacity in 'Eureka' lemon (Citrus limon (L.) Burm. f.), Sci Hortic, 248, 70-80. 


\section{Table $\mathbf{1}$ (on next page)}

PCR primers used in this study 
Table1. PCR primers used in this study

\begin{tabular}{|c|c|c|}
\hline Genes & Forward & Reverse \\
\hline CitActin & CCAAGCAGCATGAAGATCAA & ATCTGCTGGAAGGTGCTGAG \\
\hline \multicolumn{3}{|c|}{ Chlorophyll metabolism related genes } \\
\hline CitCHLH & CGATGTTCGTGAAGCAGCAACTC & TTGGAATGTGGCGTCTGCTGTGC \\
\hline CitNYC & GGCACGGTTTTCCTTTACAGATG & TGTTGTAGTTCTGACGCTTTCTG \\
\hline CitChlase & GTGGGATTGTGGTGGCGTTTCT & ACTTTTACATGAGTTGTCGTAAGC \\
\hline CitPao & CAGCACACCCTCAAGTGTTCATC & AAACAAAGGGAATACTGAGGAAAC \\
\hline CitSGR & CAACTGTTGCTTTCCTCCAATGAG & TAAAACCCCACCAATACTTTG \\
\hline CitCAB1 & ATCCATTGGGTTTGGCTGATGAC & AACTCTTATCAACCGAAGCTCACT \\
\hline CitCAB2 & CCGTCTGGCTATGTTCTCCATGT & AGATGAAACAGACACCATCAAGTC \\
\hline CitRCCR & AGTGTGCTTGTGGAGGGAGACAT & AATTAAGGTCAATCAACTCCAATTC \\
\hline \multicolumn{3}{|c|}{ Carotenoid metabolism related genes } \\
\hline CitPSY1 & CGTTGATGGGCCTAATGCTT & ACCTGGACTCCCACCTGTCTAA \\
\hline CitPSY2 & GATTCCTCAGCTTCTGCTC & CTTGCTCTTGTAATTTGCTCT \\
\hline CitPDS & TGGCAACCCCCCAGAGA & CACCCAGTGACTGAATGTGTT \\
\hline CitZDS & AAAGGCACTTGTTGATCCTGATG & ACCAATCAGAGAAGCTTATACTATCCA \\
\hline CitCRISO & AAAGACACACCGGCGGTATC & CGAGGCATTGGCCCATAG \\
\hline CitLCYb2 & CCTTGGCTCAACCAGGATGA & ACCCATTCCACACTTTCTGATGA \\
\hline CitLCYe & AAGGTGTGTCGAGTCAGGTGTTT & CCACTGGTAGATTCCGTAATGCT \\
\hline CitCHYB & GCGGCTCACCAGCTTCAC & CCGAGAAAGAGCCCATATGG \\
\hline CitZEP & CTAAAGAGCTATGAGAGAGCTAGGAGACT & CACTGCGGCCGATCTTG \\
\hline CitVDE & CAAAGACTTCAATGGGAAGTGGTA & TGGCAATCAAAAGTATCGAAGGA \\
\hline CitCCD1 & TTCATGGTCTGCGCATCAA & GACGTGAAGTCCTCACAAAACG \\
\hline CitNCED1 & AACCCGTCTGCCAGAACCTT & GTTGGCTCCGTTTCTGACGTA \\
\hline CitNCED2 & GGTGCCAACCCATTATTCGA & GCCGTCACCGTCAAAGAAAT \\
\hline CitNCED3 & GCTTCCGTTTGTGGCCTACTT & ATTGACCCGGCATTTTTATGTG \\
\hline
\end{tabular}

2 


\section{Table 2 (on next page)}

Effect of pre-harvest bagging and post-harvest ethylene de-greening treatments on individual carotenoid contents

All values were the mean $\pm S D$ of three independent biological replicates measurements. The different small letters in the same row indicated significant differences at the 0.05 level, and ND represented not detected. 

carotenoid contents

\begin{tabular}{|c|c|c|c|}
\hline $\mathrm{Carotenoids}_{\text {Groups }}^{\text {Groups }}$ & Control $\left(\mu \mathrm{g} \mathrm{g}^{-1}\right)$ & Bagging ( $\left.\mu \mathrm{g} \mathrm{g}^{-1}\right)$ & Ethylene $\left(\mu \mathrm{g} \mathrm{g}^{-1}\right)$ \\
\hline$\alpha$-carotene & $0.33 \pm 0.019^{\mathrm{a}}$ & $0.06 \pm 0.001^{b}$ & $0.06 \pm 0.003^{b}$ \\
\hline$\alpha$-cryptoxanthin & $0.22 \pm 0.018^{\mathrm{a}}$ & $0.17 \pm 0.000^{\mathrm{b}}$ & $0.17 \pm 0.000^{\mathrm{b}}$ \\
\hline$\beta$-carotene & $3.45 \pm 0.234^{\mathrm{a}}$ & $0.09 \pm 0.014^{\mathrm{b}}$ & $0.24 \pm 0.016^{b}$ \\
\hline$\beta$-apoline aldehyde & $0.11 \pm 0.004^{\mathrm{a}}$ & $0.06 \pm 0.003^{b}$ & $0.06 \pm 0.005^{\mathrm{bc}}$ \\
\hline$\beta$ - cryptoxanthin & $3.10 \pm 0.26^{\mathrm{a}}$ & $0.07 \pm 0.041^{\mathrm{b}}$ & $0.27 \pm 0.078^{\mathrm{b}}$ \\
\hline$\gamma$ - carotene & $0.34 \pm 0.019^{\mathrm{a}}$ & $0.17 \pm 0.007^{\mathrm{b}}$ & $0.15 \pm 0.006^{\mathrm{c}}$ \\
\hline$\varepsilon$ - carotene & ND & ND & ND \\
\hline phytoene & $3.71 \pm 0.433^{\mathrm{c}}$ & $120.53 \pm 9.305^{\mathrm{a}}$ & $14.17 \pm 2.016^{\mathrm{b}}$ \\
\hline Hexahydro lycopene & ND & ND & ND \\
\hline lutein & $15.62 \pm 0.785^{\mathrm{a}}$ & $0.10 \pm 0.022^{\mathrm{c}}$ & $1.73 \pm 0.148^{b}$ \\
\hline neoxanthin & $0.26 \pm 0.006^{\mathrm{a}}$ & ND & $0.03 \pm 0.005^{\mathrm{b}}$ \\
\hline zeaxanthin & $2.55 \pm 0.231^{\mathrm{a}}$ & $1.36 \pm 0.169^{c}$ & $2.00 \pm 0.238^{b}$ \\
\hline lycopene & $0.65 \pm 0.023^{\mathrm{a}}$ & $0.69 \pm 0.051^{\mathrm{a}}$ & $0.64 \pm 0.039^{\mathrm{a}}$ \\
\hline violaxanthin & $2.07 \pm 0.339^{\mathrm{a}}$ & $0.42 \pm 0.050^{\mathrm{c}}$ & $0.76 \pm 0.050^{\mathrm{b}}$ \\
\hline antheraxanthin & $0.27 \pm 0.021^{\mathrm{a}}$ & $0.01 \pm 0.001^{\mathrm{c}}$ & $0.065 \pm 0.006^{\mathrm{b}}$ \\
\hline astaxanthin & ND & ND & ND \\
\hline capsanthin & ND & $0.12 \pm 0.009^{\mathrm{a}}$ & $0.03 \pm 0.000^{\mathrm{b}}$ \\
\hline capsorubin & $0.02 \pm 0.000^{\mathrm{b}}$ & $0.02 \pm 0.000^{\mathrm{c}}$ & $0.03 \pm 0.001^{\mathrm{a}}$ \\
\hline
\end{tabular}

3 Note: all values were the mean \pm SD of three independent biological replicates measurements. The different small letters in the same row indicated

4 significant differences at the 0.05 level, and ND represented not detected.

5

6 


\section{Table 3 (on next page)}

Effect of pre-harvest bagging and post-harvest ethylene de-greening treatments on genes expression associated with carotenoid and chlorophyll metabolism

All values are the mean $\pm S D$ of three independent biological replicates measurements. The

different small letters in the same row indicate significant differences at the 0.05 level, and ND indicates not detected. 
1 Table 3. Effect of pre-harvest bagging and post-harvest ethylene de-greening treatments on genes expression 2 associated with carotenoid and chlorophyll metabolism

\begin{tabular}{|c|c|c|c|}
\hline & Control & Bagging & Ethylene \\
\hline CitCHLH & $1.31 \pm 0.13^{\mathrm{a}}$ & $1.03 \pm 0.12^{\mathrm{b}}$ & $0.56 \pm 0.08^{c}$ \\
\hline CitNYC & $0.43 \pm 0.05^{\mathrm{b}}$ & $1.08 \pm 0.16^{\mathrm{a}}$ & $0.19 \pm 0.02^{\mathrm{c}}$ \\
\hline CitChlase & $3.39 \pm 0.29^{\mathrm{a}}$ & $0.82 \pm 0.18^{b}$ & $0.40 \pm 0.05^{\mathrm{c}}$ \\
\hline CitPao & $1.58 \pm 0.20^{\mathrm{a}}$ & $1.13 \pm 0.15^{\mathrm{b}}$ & $0.90 \pm 0.09^{c}$ \\
\hline CitSGR & $0.75 \pm 0.12^{\mathrm{b}}$ & $1.08 \pm 0.20^{\mathrm{a}}$ & $0.59 \pm 0.09^{c}$ \\
\hline CitCAB1 & $2.86 \pm 0.45^{\mathrm{a}}$ & $1.07 \pm 0.11^{\mathrm{b}}$ & $0.46 \pm 0.06^{\mathrm{c}}$ \\
\hline CitCAB2 & $3.51 \pm 0.44^{\mathrm{a}}$ & $1.04 \pm 0.11^{\mathrm{b}}$ & $0.91 \pm 0.94^{\mathrm{c}}$ \\
\hline CitRCCR & $2.46 \pm 0.30^{\mathrm{a}}$ & $1.27 \pm 0.44^{\mathrm{c}}$ & $1.90 \pm 0.31^{b}$ \\
\hline CitPSY1 & $0.70 \pm 0.10^{\mathrm{b}}$ & $1.05 \pm 0.07^{\mathrm{a}}$ & $0.57 \pm 0.08^{c}$ \\
\hline CitPSY2 & $3.84 \pm 0.40^{\mathrm{a}}$ & $0.76 \pm 0.19^{c}$ & $2.74 \pm 0.39^{b}$ \\
\hline CitPDS & $1.61 \pm 0.17^{\mathrm{a}}$ & $1.03 \pm 0.12^{\mathrm{b}}$ & $0.81 \pm 0.08^{c}$ \\
\hline CitZDS & $2.48 \pm 0.46^{\mathrm{a}}$ & $1.11 \pm 0.16^{\mathrm{b}}$ & $1.07 \pm 0.14^{\mathrm{b}}$ \\
\hline CitCRTISO & $1.00 \pm 0.19^{\mathrm{a}}$ & $1.12 \pm 0.27^{\mathrm{a}}$ & $0.78 \pm 0.12^{b}$ \\
\hline CitLCYb2 & $2.66 \pm 0.27^{\mathrm{a}}$ & $1.40 \pm 0.45^{\mathrm{b}}$ & $0.73 \pm 0.13^{\mathrm{c}}$ \\
\hline CitCHYB & $1.60 \pm 0.09^{\mathrm{a}}$ & $1.44 \pm 0.37^{\mathrm{a}}$ & $0.73 \pm 0.18^{b}$ \\
\hline CitZEP & $3.02 \pm 0.65^{\mathrm{a}}$ & $1.63 \pm 0.59^{\mathrm{b}}$ & $1.05 \pm 0.33^{\mathrm{c}}$ \\
\hline CitVDE & $0.63 \pm 0.104^{\mathrm{b}}$ & $0.82 \pm 0.15^{\mathrm{a}}$ & $0.51 \pm 0.09^{c}$ \\
\hline CitCCD1 & $0.97 \pm 0.17^{\mathrm{a}}$ & $0.93 \pm 0.13^{\mathrm{ab}}$ & $0.77 \pm 0.18^{b}$ \\
\hline CitNCED1 & $0.69 \pm 0.13^{\mathrm{b}}$ & $0.85 \pm 0.18^{\mathrm{a}}$ & $0.27 \pm 0.08^{c}$ \\
\hline CitNCED2 & $1.01 \pm 0.14^{\mathrm{a}}$ & $1.08 \pm 0.15^{\mathrm{a}}$ & $0.23 \pm 0.04^{b}$ \\
\hline CitNCED3 & $0.54 \pm 0.11^{\mathrm{b}}$ & $1.53 \pm 0.72^{\mathrm{a}}$ & $0.12 \pm 0.01^{\mathrm{c}}$ \\
\hline CitLCYe & $1.71 \pm 0.27^{\mathrm{a}}$ & $1.04 \pm 0.08^{b}$ & $0.89 \pm 0.12^{\mathrm{b}}$ \\
\hline
\end{tabular}

3

Note: all values are the mean $\pm \mathrm{SD}$ of at least three independent biological replicates measurements. The different small letters in the same row indicate

4 significant differences at the 0.05 level, and ND indicates not detected. 


\section{Figure 1}

pathway of chlorophyll degradation

Red named genes are those studied in this work. Gene names are abbreviated as follows:

HEMA, Glutamy 1-tRNA reductase; GAS, 2,1-aminotransferase; HEMB, d-Aminolevulinic acid dehydratase; HEMC, porphobilinogen deaminase; HEMD, Uroporphyrinogen III synthase; HEME, Uroporphyrinogen III decarboxylase; HEMF, Coproporphyrinogen III oxidase; $\mathrm{CHLH}, \mathrm{Mg}$ chelatase; NYC, chlorophyll b reductase; CAB, chlorophyll a/b binding protein; SGR, staygreen protein; Chlase, chlorophyllase; MCS, metal quelating substance; Pao, pheophorbide a oxygenase; RCCR, real chorophyll catabolite reductase; LHCP, chlorophyll bound. 


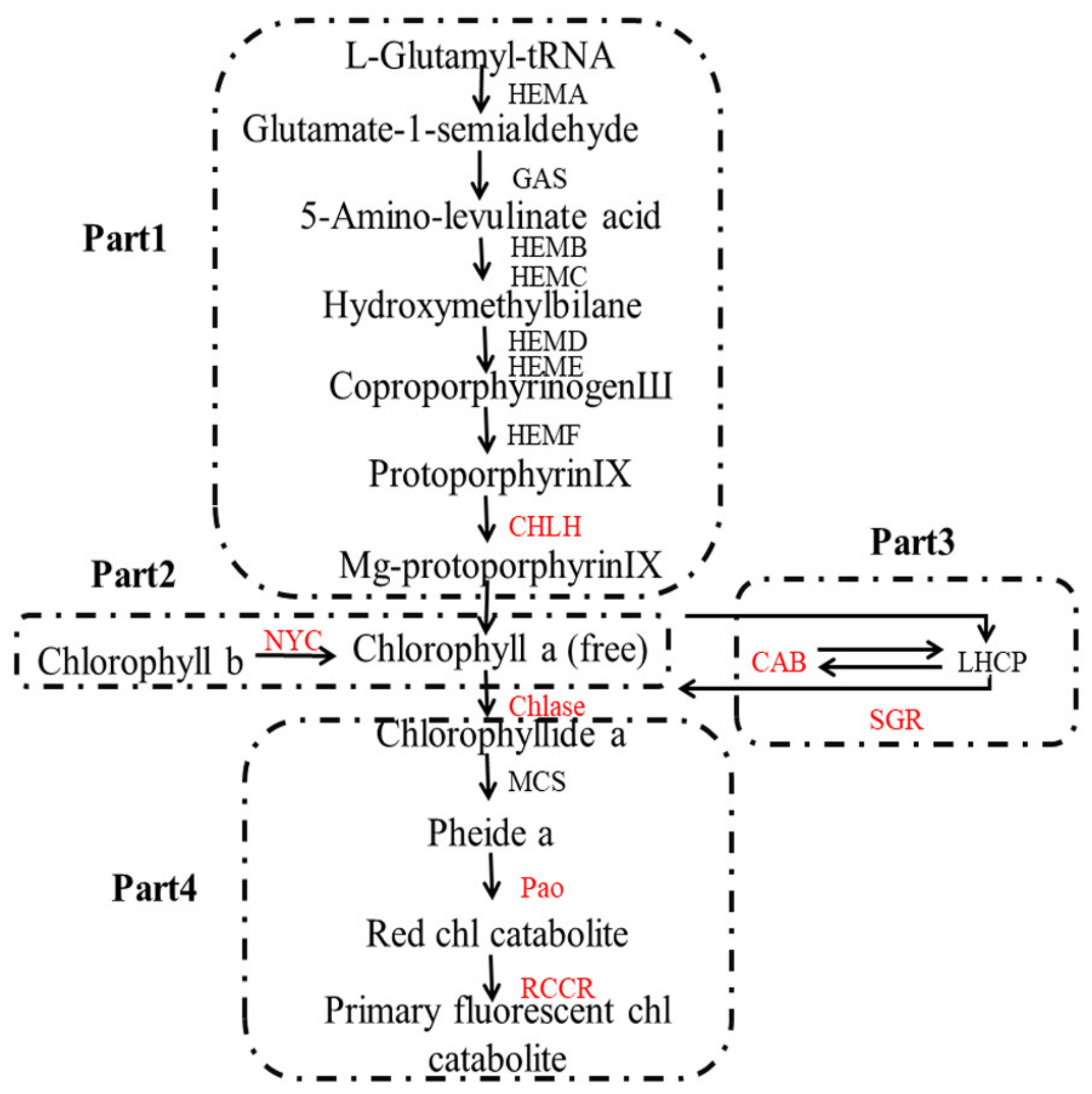




\section{Figure 2}

pathway of carotenoid biosynthesis

Red named genes are those studied in this work. Gene names are abbreviated as follows: PSY, phytoene synthase; PDS, phytoene desaturase; ZDS, $\zeta$-carotene desaturase; CRTISO, carotenoid isomerase; LCYe, lycopene $\varepsilon$-cyclase; LCYb1, lycopene $\square$-cyclase 1; LCYb2, lycopene $\square$-cyclase 2; CHYB, $\square$-carotene hydroxylase; CCDs, carotenoid cleavage dioxygenase; ZEP, zeaxanthin synthase; VDE, violaxanthin de-epoxidase; NSY, neoxanthin synthase; NCEDs, 9-cis-epoxycarotenoid dioxygenase. 


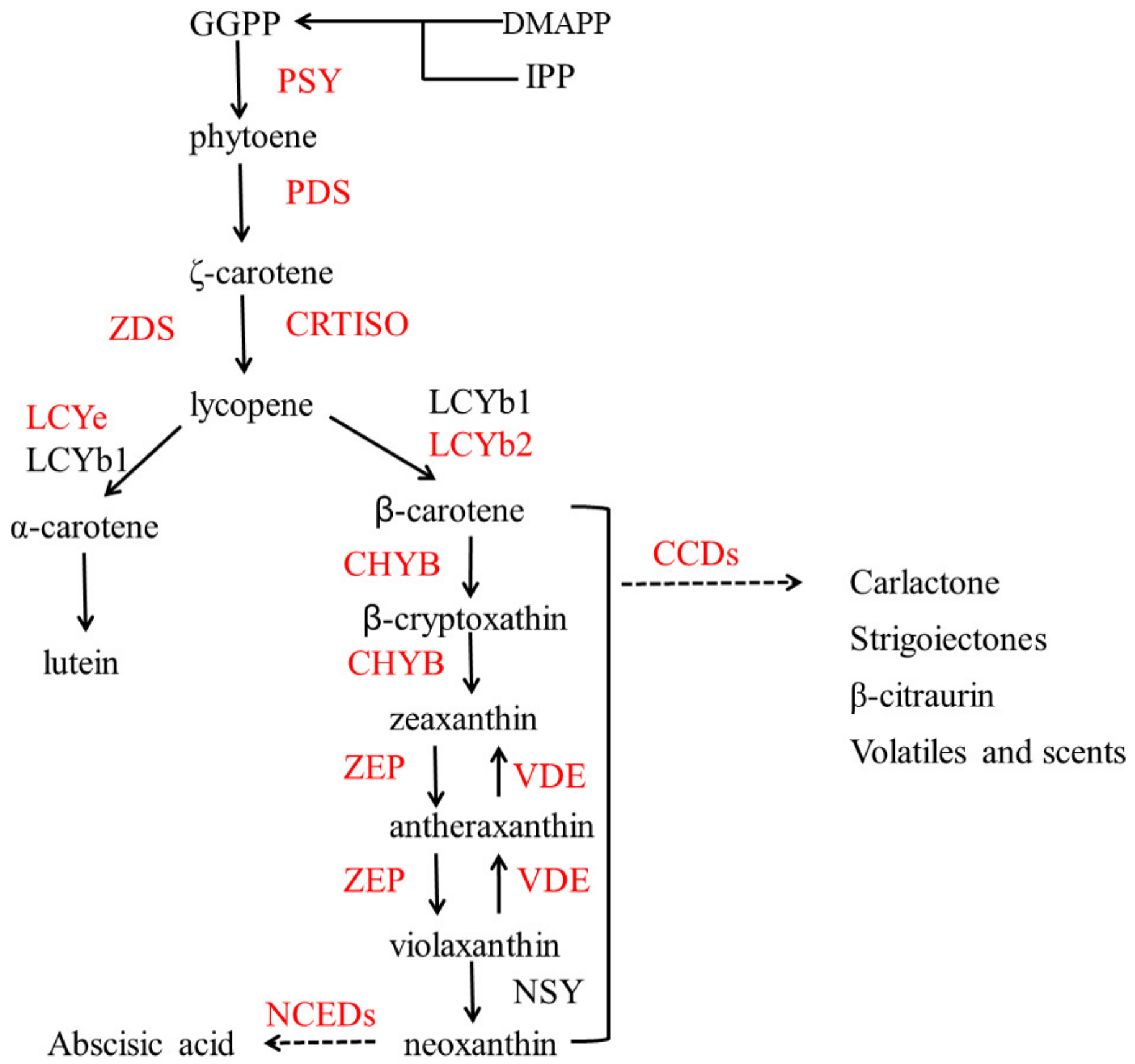


Figure 3

The picture of the lemon

\section{Control}

Bagging

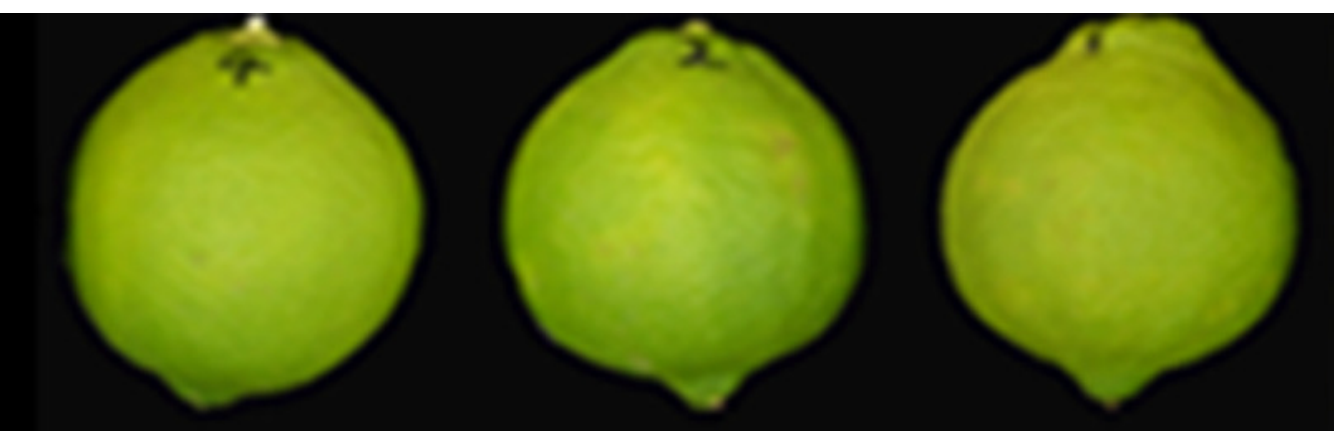

Ethylene
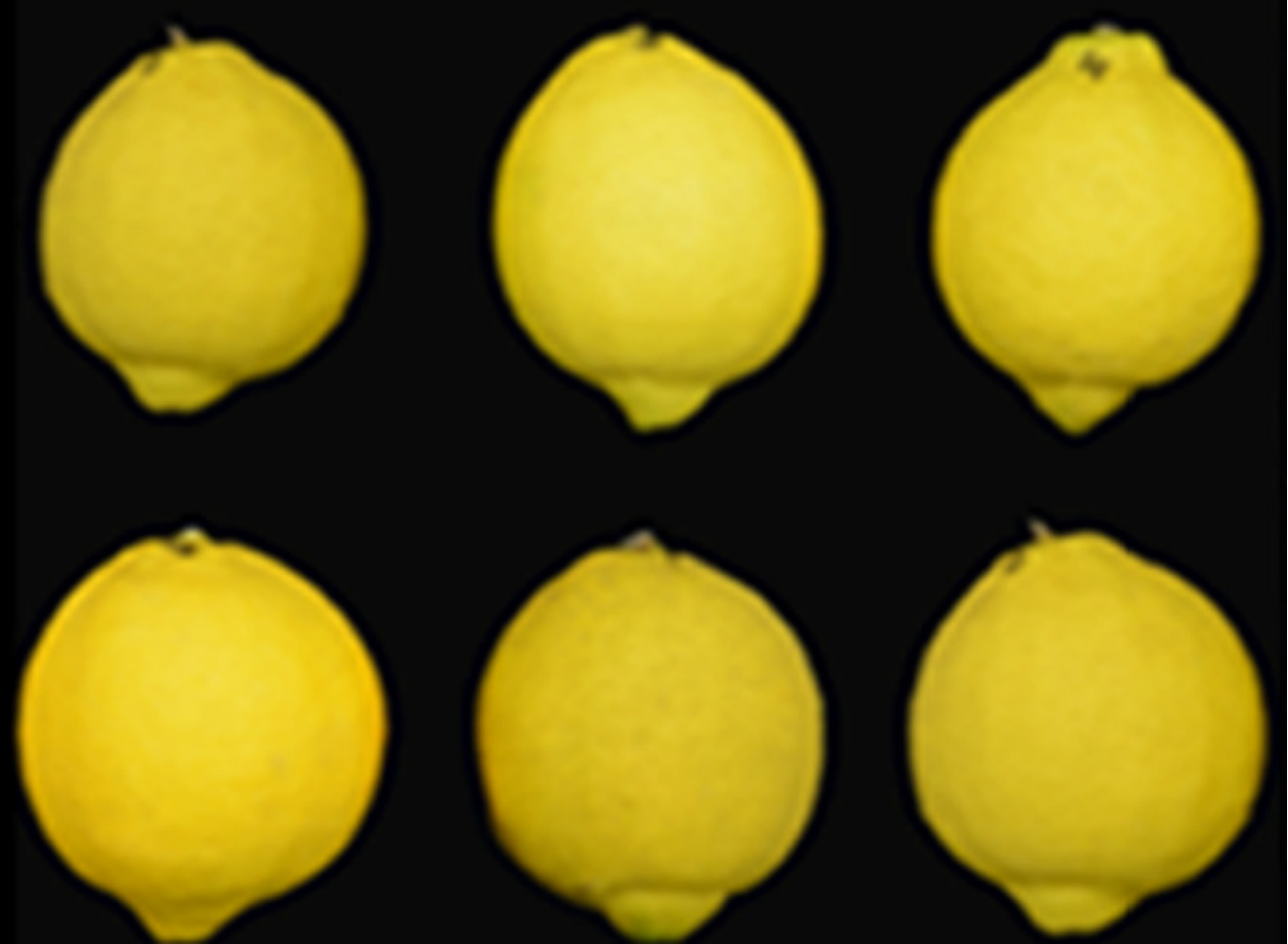


\section{Figure 4}

Effect of pre-harvest bagging and post-harvest ethylene de-greening treatments on chromatic aberration

$L^{*}$ the lightness ranging from black to white; $a^{*}$ a scale ranging from green to red; and $b^{*} a$ scale ranging from blue to yellow. Vertical bars represent standard deviations of the three independent biological replicates means. Different letters are statistically different by the Duncan's multiple range (P\0.05).

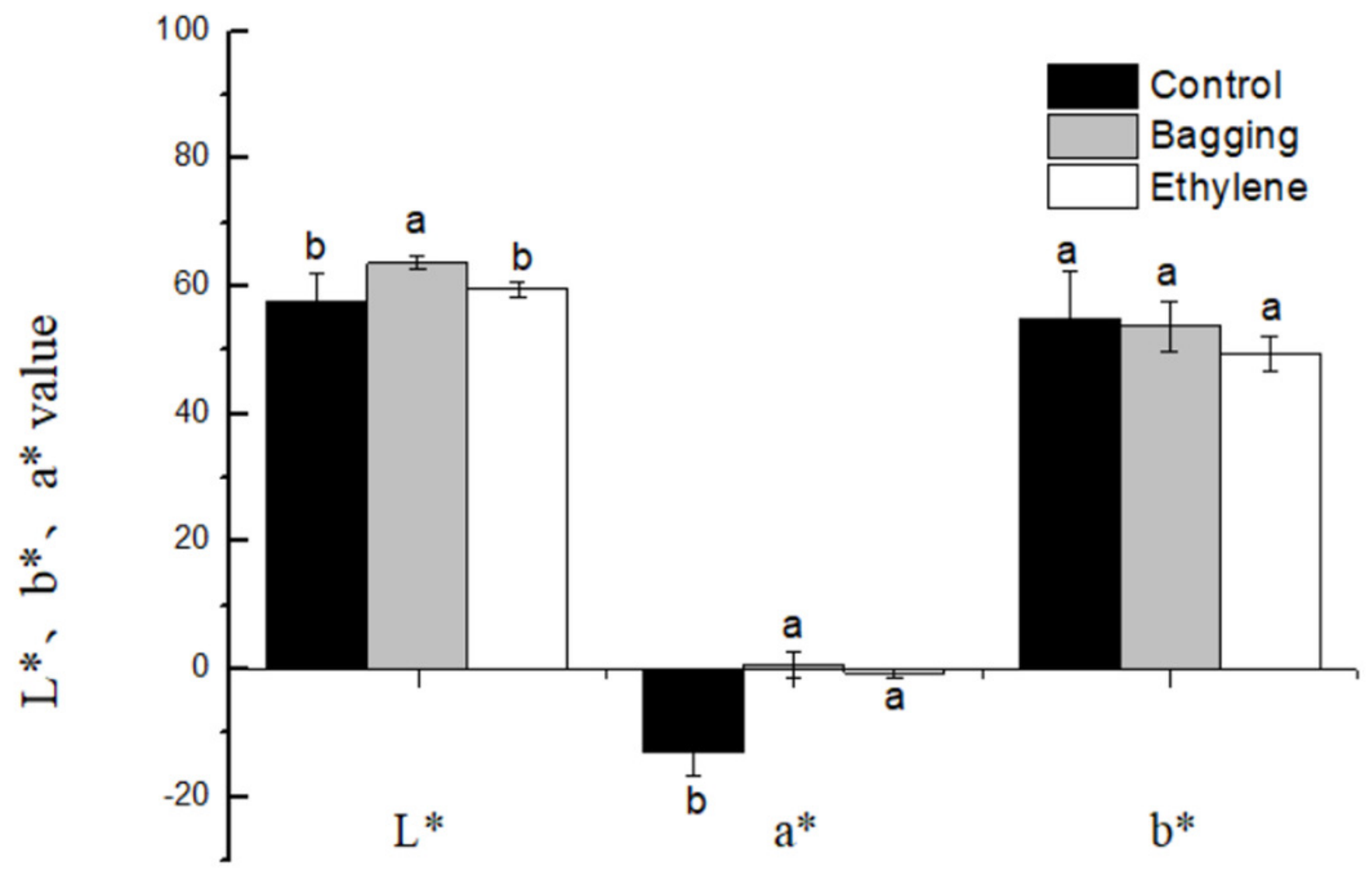




\section{Figure 5}

Effect of pre-harvest bagging and post-harvest ethylene de-greening treatments on pigment

Vertical bars represent standard deviations of the three independent biological replicates means. Different letters are statistically different by the Duncan's multiple range (P־0.05).

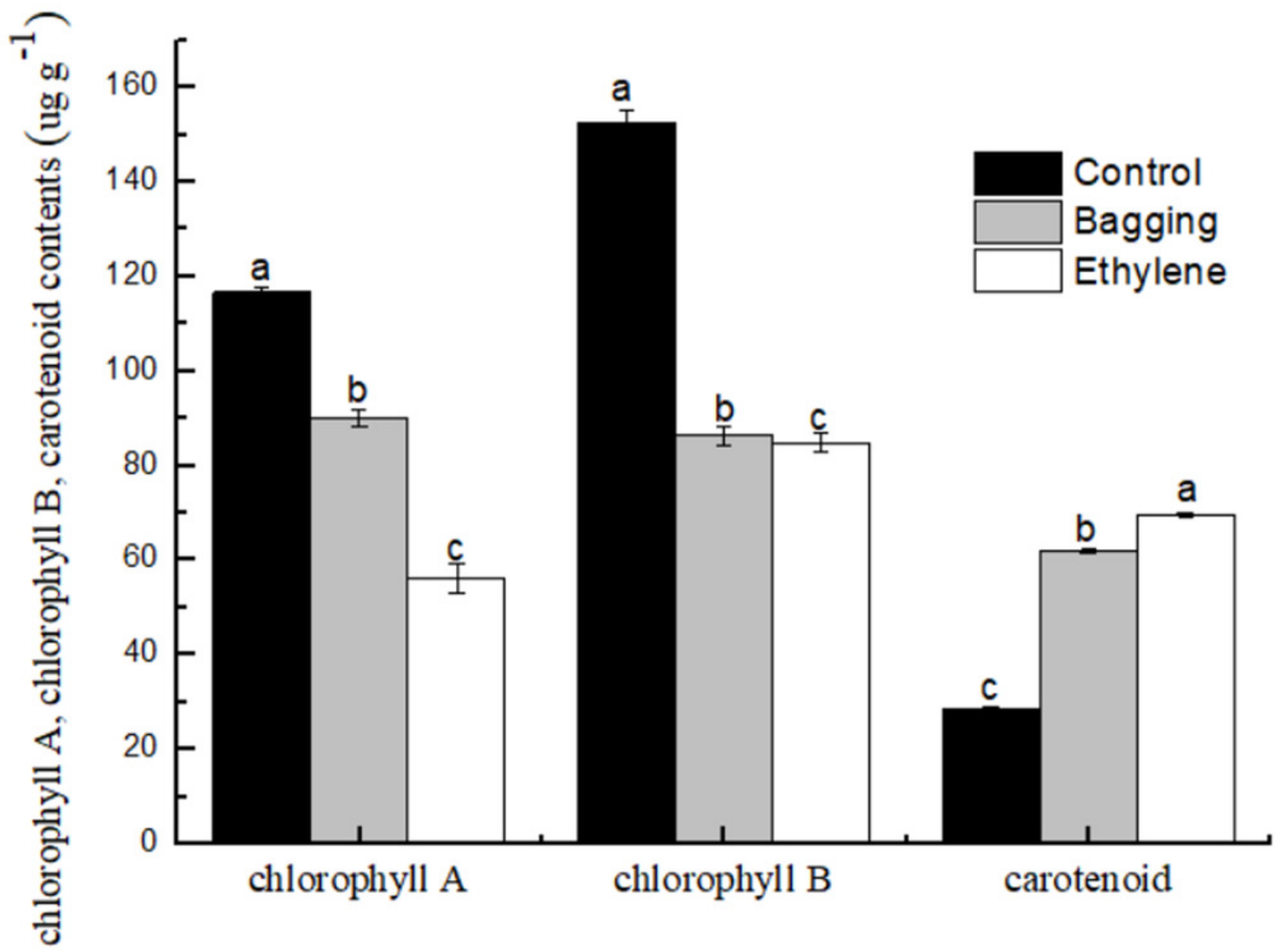


Figure 6

Heat map diagram of expression levels for chlorophyll and carotenoid degradation pathway genes analyzed by KEGG.T means Bagging, Q means control, Z means ethylene.

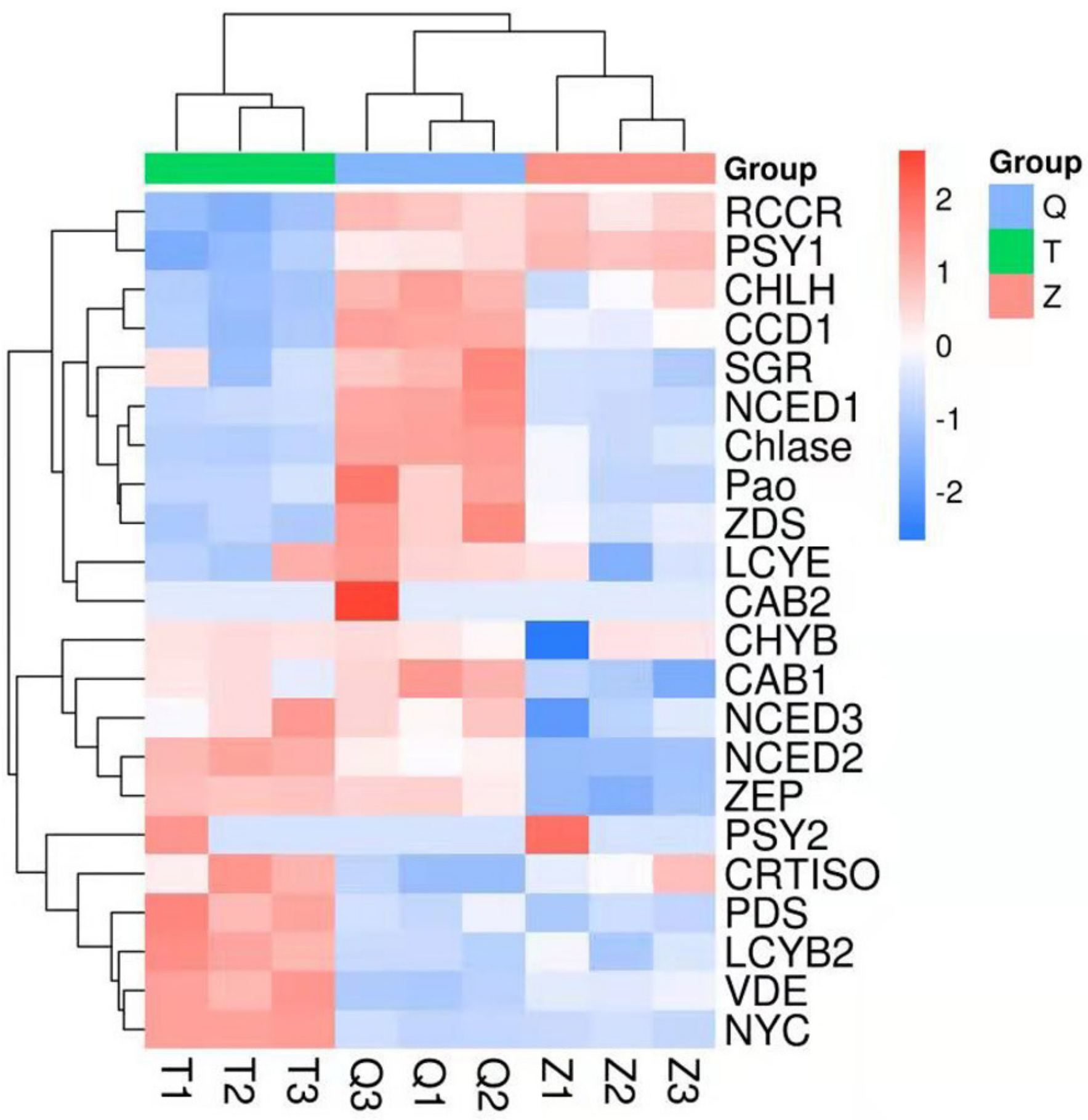

